\title{
Discrete limit theorems for the Mellin transform of the Riemann zeta-function
}

by

\section{Violeta Balinskaitė and Antanas LaurinčIKas (Vilnius)}

1. Introduction. As usual, denote by $\zeta(s), s=\sigma+i t$, the Riemann zeta-function. In analytic number theory the modified Mellin transforms $\mathcal{Z}_{k}(s)$ of powers of the function $\zeta(s)$ are considered. For $k \geq 0$ and $\sigma \geq$ $\sigma_{0}(k)>1$, the function $\mathcal{Z}_{k}(s)$ is defined by

$$
\mathcal{Z}_{k}(s)=\int_{1}^{\infty}|\zeta(1 / 2+i x)|^{2 k} x^{-s} d x
$$

In view of the Mellin inversion formula the function $\mathcal{Z}_{k}(s)$ is very useful for the investigation of power moments

$$
\int_{0}^{T}|\zeta(1 / 2+i t)|^{2 k} d t
$$

of the Riemann zeta-function.

This paper is devoted to the asymptotic behavior of $\mathcal{Z}_{2}(s)$. We recall that $\mathcal{Z}_{2}(s)$ is a meromorphic function having a pole at $s=1$ of order five, simple poles at $s=1 / 2 \pm i \sqrt{\lambda_{j}-1 / 4}$, where $\left\{\lambda_{j}\right\} \cup\{0\}$ is the discrete spectrum of the non-Euclidean Laplacian acting on automorphic forms for the full modular group, and poles at $s=\varrho / 2$ for any complex zero $\varrho$ of the Riemann zeta-function.

This as well as estimates and mean-square estimates for $\mathcal{Z}_{2}(s)$ were obtained in [7]-[11].

The idea of applying probabilistic methods in the theory of functions belongs to H. Bohr and B. Jessen [3], [4]. Later, Bohr-Jessen's theory was developed by many authors; for history and results see [12], [14].

2000 Mathematics Subject Classification: 11M06, 44A15.

Key words and phrases: limit theorem, Mellin transform, Riemann zeta-function, weak convergence.

Partially supported by Lithuanian Foundation of Studies and Science. 
The first attempt to study the function $\mathcal{Z}_{2}(s)$ in this direction was made in [13]. To state the theorems of [13] we need some notation. Let meas $\{A\}$ denote the Lebesgue measure of a measurable set $A \subset \mathbb{R}$, and let, for $T>0$,

$$
\nu_{T}^{t}(\ldots)=\frac{1}{T} \operatorname{meas}\{t \in[0, T]: \ldots\},
$$

where the dots stand for a condition satisfied by $t$. Here the $t$ in $\nu_{T}^{t}$ only indicates that the measure is taken over $t \in[0, T]$. Denote by $\mathcal{B}(S)$ the class of Borel subsets of the space $S$.

Theorem 1. Let $7 / 8<\sigma<1$. Then on $(\mathbb{C}, \mathcal{B}(\mathbb{C}))$ there exists a probability measure $P_{\mathbb{C}, \sigma}$ such that the probability measure

$$
\nu_{T}^{t}\left(\mathcal{Z}_{2}(\sigma+i t) \in A\right), \quad A \in \mathcal{B}(\mathbb{C}),
$$

converges weakly to $P_{\mathbb{C}, \sigma}$ as $T \rightarrow \infty$.

Now let $G$ be a region on the complex plane. Denote by $H(G)$ the space of analytic functions on $G$ equipped with the topology of uniform convergence on compacta. Let $\widehat{D}=\{s \in \mathbb{C}: 7 / 8<\sigma<1\}$.

TheOrEm 2. On $(H(\widehat{D}), \mathcal{B}(H(\widehat{D})))$ there exists a probability measure $P_{H}$ such that the probability measure

$$
\nu_{T}^{\tau}\left(\mathcal{Z}_{2}(s+i \tau) \in A\right), \quad A \in \mathcal{B}(H(\widehat{D})),
$$

converges weakly to $P_{H}$ as $T \rightarrow \infty$.

Note that Theorems 1 and 2 are valid in a comparatively narrow region. This follows from known mean-square estimates for the function $\mathcal{Z}_{2}(s)$.

Theorems 1 and 2 are of so called continuous character, because the imaginary part $t$ or $\tau$ of shifts varies continuously in $[0, T]$. The aim of this paper is to obtain discrete limit theorems for $\mathcal{Z}_{2}(s)$, when the imaginary part of shifts in the definition of probability measures takes values in some arithmetical progression. For $N \in \mathbb{N} \cup\{0\}$, let

$$
\mu_{N}(\ldots)=\frac{1}{N+1} \sum_{0 \leq m \leq N} 1,
$$

where the dots stand for a condition satisfied by $m$. Let $h>0$ be a fixed number. Define a probability measure by

$$
P_{N, \sigma}(A)=\mu_{N}\left(\mathcal{Z}_{2}(\sigma+i m h) \in A\right), \quad A \in \mathcal{B}(\mathbb{C}) .
$$

Theorem 3. Let $\sigma>5 / 6$. Then on $(\mathbb{C}, \mathcal{B}(\mathbb{C}))$ there exists a probability measure $P_{\sigma}$ such that $P_{N, \sigma}$ converges weakly to $P_{\sigma}$ as $N \rightarrow \infty$.

Now let $D=\{s \in \mathbb{C}: 5 / 6<\sigma<1\}$ and

$$
P_{N}(A)=\mu_{N}\left(\mathcal{Z}_{2}(s+i m h) \in A\right), \quad A \in \mathcal{B}(H(D)) .
$$


Theorem 4. On $(H(D), \mathcal{B}(H(D)))$ there exists a probability measure $P$ such that $P_{N}$ converges weakly to $P$ as $N \rightarrow \infty$.

For the proof of Theorems 3 and 4 we use a new mean-square estimate for $\mathcal{Z}_{2}(s)$. Therefore, these theorems are valid in wider regions than Theorems 1 and 2. Clearly, Theorem 4 is also valid in the half-plane $\{s \in \mathbb{C}: \sigma>1\}$.

To prove limit theorems for Dirichlet series or their integral analogues (Laplace or Mellin transforms) usually the method of Fourier or characteristic transforms as well as the Prokhorov theory (tightness and relative compactness of families of probability measures) are applied. However, the machinery of discrete limit theorems is quite different from that of the continuous case. In continuous theorems one deals with mathematical objects given by integrals, while in the case of discrete limit theorems, trigonometric and other sums appear. Therefore, discrete theorems are more complicated, they depend on a chosen discrete set used to define the relevant probability measures. Note that in the discrete case the Gallagher lemma (Lemma 1.4 from [5]), which relates continuous and discrete mean-square estimates, is very useful. On the other hand, the discrete results are more convenient for applications. For example, the discrete universality for zeta-functions is used to estimate complicated integrals over analytic curves [2]. Theorems 3 and 4 are the first step in this direction. In future we will identify the limit measures in these theorems. This would open the possibility to consider the universality of the Mellin transforms.

2. A limit theorem on a torus. Let $\gamma=\{s \in \mathbb{C}:|s|=1\}$ be the unit circle on the complex plane. For $a>1$, define

$$
\Omega_{a}=\prod_{u \in[1, a]} \gamma_{u}
$$

where $\gamma_{u}=\gamma$ for all $u \in[1, a]$. Since $\gamma$ is a compact, by the Tikhonov theorem the torus $\Omega_{a}$ is a compact topological Abelian group. Note that the Tikhonov theorem holds for products over any set of indices [16].

TheOREM 5. On $\left(\Omega_{a}, \mathcal{B}\left(Q_{a}\right)\right)$ there exists a probability measure $Q_{a}$ such that the probability measure

$$
Q_{N, a}(A):=\mu_{N}\left(\left(u^{i m h}: u \in[1, a]\right) \in A\right), \quad A \in \mathcal{B}\left(\Omega_{a}\right),
$$

converges weakly to $Q_{a}$ as $N \rightarrow \infty$.

Proof. Let $\mathbb{Z}$ denote the set of all integers. The dual group of $\Omega_{a}$ is

$$
\bigoplus_{u \in[1, a]} \mathbb{Z}_{u}
$$


where $\mathbb{Z}_{u}=\mathbb{Z}$ for all $u \in[1, a]$. An element $\underline{k}=\left\{k_{u}: u \in[1, a]\right\} \in$ $\bigoplus_{u \in[1, a]} \mathbb{Z}_{u}$, where only a finite number of integers $k_{u}$ are non-zero, acts on $\Omega_{a}$ by

$$
\underline{x} \mapsto \underline{x}^{\underline{k}}=\prod_{u \in[1, a]} x_{u}^{k_{u}},
$$

where $\underline{x}=\left\{x_{u}: x_{u} \in \gamma, u \in[1, a]\right\}$. Therefore, the Fourier transform $g_{N}(\underline{k})$ of the probability measure $Q_{N}$ is

$$
\begin{aligned}
g_{N}(\underline{k}) & =\int_{\Omega} \prod_{u \in[1, a]} x_{u}^{k_{u}} d Q_{N, a}=\frac{1}{N+1} \sum_{m=0}^{N} \prod_{u \in[1, a]} e^{i m h k_{u} \log u} \\
& =\frac{1}{N+1} \sum_{m=0}^{N} \exp \left\{i m h \sum_{u \in[1, a]} k_{u} \log u\right\}
\end{aligned}
$$

where only a finite number of integers $k_{u}$ are non-zero. Since

$$
\exp \left\{i h \sum_{u \in[1, a]} k_{u} \log u\right\}=1
$$

if and only if there exists $r \in \mathbb{Z}$ such that

we deduce that

$$
\sum_{u \in[1, a]} k_{u} \log u=\frac{2 \pi r}{h},
$$

$$
g_{N}(\underline{k})=\left\{\begin{array}{l}
1 \text { if } \sum_{u \in[1, a]} k_{u} \log u=2 \pi r / h \text { for some } r \in \mathbb{Z} \\
\frac{1}{N+1} \frac{1-\exp \left\{i(N+1) h \sum_{u \in[1, a]} k_{u} \log u\right\}}{1-\exp \left\{i h \sum_{u \in[1, a]} k_{u} \log u\right\}} \text { otherwise. }
\end{array}\right.
$$

Therefore,

(1) $\lim _{N \rightarrow \infty} g_{N}(\underline{k})= \begin{cases}1 & \text { if } \sum_{u \in[1, a]} k_{u} \log u=2 \pi r / h \text { for some } r \in \mathbb{Z}, \\ 0 & \text { otherwise. }\end{cases}$

Thus, by the continuity theorem for probability measures on locally compact topological groups (see, for example, [6, Theorem 1.4.2]), the probability measure $Q_{N, a}$ converges weakly to a probability measure $Q_{a}$ with the Fourier transform given by the right-hand side of (1). The theorem is proved.

3. Limit theorems for integrals over a finite interval. Let $a>1$. In this section we will prove limit theorems for the integral

$$
\mathcal{Z}_{2, a, y}(s):=\int_{1}^{a}|\zeta(1 / 2+i x)|^{4} v(x, y) x^{-s} d x
$$

where, for $y \geq 1$,

$$
v(x, y)=\exp \left\{-(x / y)^{\sigma_{1}}\right\}
$$

with fixed $\sigma_{1}>1 / 2$. 
Theorem 6. On $(\mathbb{C}, \mathcal{B}(\mathbb{C}))$ there exists a probability measure $P_{\sigma, a, y}$ such that the probability measure

$$
P_{N, \sigma, a, y}(A):=\mu_{N}\left(\mathcal{Z}_{2, a, y}(\sigma+i m h) \in A\right), \quad A \in \mathcal{B}(\mathbb{C}),
$$

converges weakly to $P_{\sigma, a, y}$ as $N \rightarrow \infty$.

Proof. For $y_{x} \in \Omega_{a}$ let

$\widehat{y}_{x}= \begin{cases}y_{x} & \text { if } y_{x} \text { is measurable over }[1, a], \\ f(x) & \text { otherwise, where } f \text { is any measurable function defined on }[1, a] .\end{cases}$ Define a function $h_{\sigma, a, y}: \Omega_{a} \rightarrow \mathbb{C}$ by the formula

$$
h_{\sigma, a, y}\left(\left\{y_{x}: x \in[1, a]\right\}\right)=\int_{1}^{a}|\zeta(1 / 2+i x)|^{4} v(x, y) x^{-\sigma} \widehat{y}_{x}^{-1} d x .
$$

The definition of $\widehat{y}_{x}$ and the Lebesgue theorem show that the function $h_{\sigma, a, y}$ is continuous. Moreover,

$$
h_{\sigma, a, y}\left(\left\{x^{i m h}: x \in[1, a]\right\}\right)=\int_{1}^{a}|\zeta(1 / 2+i x)|^{4} v(x, y) x^{-\sigma-i m h} d x .
$$

Hence $P_{N, \sigma, a, y}=Q_{N, a} h_{\sigma, a, y}^{-1}$. Therefore, Theorem 5 and Theorem 5.1 of [1] show that $P_{N, \sigma, a, y}$ converges weakly to $Q_{a} h_{\sigma, a, y}^{-1}$ as $N \rightarrow \infty$.

TheOREM 7. On $(H(D), \mathcal{B}(H(D)))$ there exists a probability measure $P_{a, y}$ such that the probability measure

$$
P_{N, a, y}(A):=\mu_{N}\left(\mathcal{Z}_{2, a, y}(s+i m h) \in A\right), \quad A \in \mathcal{B}(H(D)),
$$

converges weakly to $P_{a, y}$ as $N \rightarrow \infty$.

Proof. Consider the function $h_{a, y}: \Omega_{a} \rightarrow H(D)$ given by the formula

$$
h_{a, y}\left(\left\{y_{x}: x \in[1, a]\right\}\right)=\int_{1}^{a}|\zeta(1 / 2+i x)|^{4} v(x, y) x^{-s} \widehat{y}_{x}^{-1} d x .
$$

Then the function $h_{a, y}$ is continuous, and

$$
h_{a, y}\left(\left\{x^{i m h}: x \in[1, a]\right\}\right)=\int_{1}^{a}|\zeta(1 / 2+i x)|^{4} v(x, y) x^{-s-i m h} d x .
$$

This shows that $P_{N, a, y}=Q_{N, a} h_{a, y}^{-1}$. Hence, in view of Theorem 5 and Theorem 5.1 of [1], the assertion follows.

4. Limit theorems for absolutely convergent integrals. In this section we will consider the function

$$
\mathcal{Z}_{2, y}(s)=\int_{1}^{\infty}|\zeta(1 / 2+i x)|^{4} v(x, y) x^{-s} d x .
$$


Lemma 8. The integral defining $\mathcal{Z}_{2, y}(s)$ converges absolutely for $\sigma>1 / 2$. Proof. For $y \geq 1$ and $\sigma_{1}>1 / 2$, let

$$
l_{y}(s)=\frac{s}{\sigma_{1}} \Gamma\left(\frac{s}{\sigma_{1}}\right) y^{s} .
$$

Define

$$
a_{y}(x)=\frac{1}{2 \pi i} \int_{\sigma_{1}-i \infty}^{\sigma_{1}+i \infty}|\zeta(1 / 2+i x)|^{4} \frac{l_{y}(s)}{s x^{s}} d s .
$$

Since $\Gamma(\sigma+i t) \ll e^{-\pi|t| / 2}|t|^{\sigma-1 / 2}$, we obtain

$$
a_{y}(x) \ll|\zeta(1 / 2+i x)|^{4} x^{-\sigma_{1}} \int_{-\infty}^{\infty}\left|l_{y}\left(\sigma_{1}+i t\right)\right| d t \ll_{y}|\zeta(1 / 2+i x)|{ }^{4} x^{-\sigma_{1}} .
$$

Using the well-known estimate

$$
\int_{0}^{T}|\zeta(1 / 2+i t)|^{4} d t \ll T \log ^{4} T,
$$

we find that, for $\sigma>1 / 2$,

$$
\begin{aligned}
\int_{1}^{\infty} \frac{a_{y}(x)}{x^{\sigma}} d x & \ll y \int_{1}^{\infty} x^{-\sigma-\sigma_{1}} d\left(\int_{0}^{x}|\zeta(1 / 2+i t)|^{4} d t\right) \\
& \left.\ll y x^{1-\sigma-\sigma_{1}} \log ^{4} x\right|_{1} ^{\infty}+\left(\sigma+\sigma_{1}\right) \int_{1}^{\infty} x^{-\sigma-\sigma_{1}} \log ^{4} x d x<\infty .
\end{aligned}
$$

The Mellin formula

$$
\frac{1}{2 \pi i} \int_{b-i \infty}^{b+i \infty} \Gamma(s) a^{-s} d s=e^{-a}
$$

with positive $a$ and $b$ together with the definitions of $a_{y}(x)$ and $v(x, y)$ yields

$$
a_{y}(x)=|\zeta(1 / 2+i x)|^{4} v(x, y) .
$$

By (2) this proves the lemma.

Theorem 9. Let $\sigma>1 / 2$. Then on $(\mathbb{C}, \mathcal{B}(\mathbb{C}))$ there exists a probability measure $P_{\sigma, y}$ such that the probability measure

$$
P_{N, \sigma, y}(A):=\mu_{N}\left(\mathcal{Z}_{2, y}(\sigma+i t) \in A\right), \quad A \in \mathcal{B}(\mathbb{C}),
$$

converges weakly to $P_{\sigma, y}$ as $N \rightarrow \infty$.

Proof. By Theorem 6, $P_{N, \sigma, a, y}$ converges weakly to $P_{\sigma, a, y}$ as $N \rightarrow \infty$. We will show that the family $\left\{P_{\sigma, a, y}\right\}$ of probability measures is tight for fixed $\sigma$ and $y$. 
Define, on a certain probability space $(\Omega, \mathcal{B}(\Omega), \mathbb{P})$, a random variable $\theta_{N}$ by the distribution law

$$
\mathbb{P}\left(\theta_{N}=h m\right)=\frac{1}{N+1}, \quad m=0, \ldots, N .
$$

Further, let

$$
X_{N, a, y}(\sigma)=\mathcal{Z}_{2, a, y}\left(\sigma+i \theta_{N}\right) .
$$

Then Theorem 6 implies

$$
X_{N, a, y}(\sigma) \underset{N \rightarrow \infty}{\stackrel{\mathcal{D}}{\longrightarrow}} X_{a, y}(\sigma),
$$

where $\underset{N \rightarrow \infty}{\stackrel{\mathcal{D}}{\longrightarrow}}$ means convergence in distribution, and $X_{a, y}(\sigma)$ is a complexvalued random variable with distribution $P_{\sigma, a, y}$. Let $M>0$. Then by Chebyshev's inequality,

$$
\mathbb{P}\left(\left|X_{N, a, y}(\sigma)\right|>M\right) \leq \frac{1}{(N+1) M} \sum_{m=0}^{N}\left|\mathcal{Z}_{2, a, y}(\sigma+i m h)\right| .
$$

Since the integral defining $\mathcal{Z}_{2, y}(s)$ converges absolutely for $\sigma>1 / 2$,

$$
\sup _{a \geq 1} \limsup _{N \rightarrow \infty} \frac{1}{N+1} \sum_{m=0}^{N}\left|\mathcal{Z}_{2, a, y}(\sigma+i m h)\right| \leq R<\infty
$$

with $R=R_{\sigma, y}=\mathcal{Z}_{2, y}(\sigma)$. Now let $M=R \varepsilon^{-1}$, where $\varepsilon$ is an arbitrary positive number. Then (4) and (5) show that

$$
\limsup _{N \rightarrow \infty} \mathbb{P}\left(\left|X_{N, a, y}(\sigma)\right|>M\right) \leq \varepsilon .
$$

The function $u: \mathbb{C} \rightarrow \mathbb{R}$ given by $h(s)=|s|, s \in \mathbb{C}$, is continuous. Therefore, (3) and (6) imply the inequality

$$
\mathbb{P}\left(\left|X_{a, y}(\sigma)\right|>M\right) \leq \varepsilon .
$$

Clearly, the set $C_{\varepsilon}=\{s \in \mathbb{C}:|s| \leq M\}$ is compact, and by (7),

$$
\mathbb{P}\left(X_{a, y}(\sigma) \in C_{\varepsilon}\right) \geq 1-\varepsilon
$$

for all $a \geq 1$. Hence, by the definition of $X_{a, y}(\sigma)$,

$$
P_{\sigma, a, y}\left(C_{\varepsilon}\right) \geq 1-\varepsilon
$$

for all $a \geq 1$, and the tightness of the family $\left\{P_{\sigma, a, y}\right\}$ is proved. Then by the Prokhorov theorem [1] the family $\left\{P_{\sigma, a, y}\right\}$ is relatively compact.

By the definitions of $\mathcal{Z}_{2, a, y}(s)$ and $\mathcal{Z}_{2, y}(s)$, for $\sigma>1 / 2$,

$$
\mathcal{Z}_{2, y}(s)=\lim _{a \rightarrow \infty} \mathcal{Z}_{2, a, y}(s)
$$


uniformly in $t$. Therefore, for $\sigma>1 / 2$ and every $\varepsilon>0$,

$$
\begin{aligned}
& \lim _{a \rightarrow \infty} \limsup _{N \rightarrow \infty} \mu_{N}\left(\left|\mathcal{Z}_{2, y}(\sigma+i m h)-\mathcal{Z}_{2, a, y}(\sigma+i m h)\right| \geq \varepsilon\right) \\
\leq & \lim _{a \rightarrow \infty} \limsup _{N \rightarrow \infty} \frac{1}{\varepsilon(N+1)} \sum_{m=0}^{N}\left|\mathcal{Z}_{2, y}(\sigma+i m h)-\mathcal{Z}_{2, a, y}(\sigma+i m h)\right|=0 .
\end{aligned}
$$

Set $X_{N, y}(\sigma)=\mathcal{Z}_{2, y}\left(\sigma+i \theta_{N}\right)$. Then by (8),

$$
\lim _{a \rightarrow \infty} \limsup _{N \rightarrow \infty} \mathbb{P}\left(\left|X_{N, y}(\sigma)-X_{N, a, y}(\sigma)\right| \geq \varepsilon\right)=0 .
$$

Since the family $\left\{P_{\sigma, a, y}\right\}$ is relatively compact, there exists a subsequence $\left\{P_{\sigma, a_{1}, y}\right\}$ converging weakly to a measure $P_{\sigma, y}$ on $(\mathbb{C}, \mathcal{B}(\mathbb{C}))$ as $a_{1} \rightarrow \infty$. In other words,

$$
X_{a_{1}, y}(\sigma) \underset{a_{1} \rightarrow \infty}{\stackrel{\mathcal{D}}{\longrightarrow}} P_{\sigma, y} .
$$

Now (3) and (9) show that Theorem 4.2 of [1] can be applied. Thus

$$
X_{N, y}(\sigma) \underset{N \rightarrow \infty}{\stackrel{\mathcal{D}}{\longrightarrow}} P_{\sigma, y},
$$

and this gives the assertion of the theorem.

TheOREM 10. On $(H(D), \mathcal{B}(H(D)))$ there exists a probability measure $P_{y}$ such that the probability measure

$$
P_{N, y}(A):=\mu_{N}\left(\mathcal{Z}_{2, y}(s+i m h) \in A\right), \quad A \in \mathcal{B}(H(D)),
$$

converges weakly to $P_{y}$ as $N \rightarrow \infty$.

Proof. We start with a metric on the space $H(D)$. It is known (see, for example, [5]) that there exists a sequence $\left\{K_{n}\right\}$ of compact subsets of the strip $D$ such that

$$
D=\bigcup_{n=1}^{\infty} K_{n}
$$

$K_{n} \subset K_{n+1}, n \in \mathbb{N}$, and if $K$ is a compact subset of $D$, then $K \subseteq K_{n}$ for some $n$. For $f, g \in H(D)$, define

$$
\varrho(f, g)=\sum_{n=1}^{\infty} 2^{-n} \frac{\varrho_{n}(f, g)}{1+\varrho_{n}(f, g)}, \quad \text { where } \quad \varrho_{n}(f, g)=\sup _{s \in K_{n}}|f(s)-g(s)| \text {. }
$$

Then it is easily seen that $\varrho(f, g)$ is a metric on $H(D)$ which induces the topology of uniform convergence on compacta.

We will preserve the notation used in the proof of Theorem 9. Define

$$
Y_{N, a, y}(s)=\mathcal{Z}_{2, a, y}\left(s+i \theta_{N}\right) .
$$

Then by Theorem 5 we have

$$
Y_{N, a, y}(s) \underset{N \rightarrow \infty}{\stackrel{\mathcal{D}}{\longrightarrow}} Y_{a, y}(s),
$$


where $Y_{a, y}(s)$ is an $H(D)$-valued random element with distribution $P_{a, y}$. First, we will show that the family $\left\{P_{a, y}\right\}$ of probability measures is tight for fixed $y$. For $M_{n}>0$, similarly to the proof of Theorem 9 , we find that

$$
\limsup _{N \rightarrow \infty} \mathbb{P}\left(\sup _{s \in K_{n}}\left|Y_{N, a, y}(s)\right|>M_{n}\right) \leq R_{n} / M_{n},
$$

where $R_{n}<\infty$. For arbitrary $\varepsilon>0$, let $M_{n}=R_{n} 2^{n} \varepsilon^{-1}$. Then, using (10) and Theorem 5.1 of [1], we deduce that

$$
\mathbb{P}\left(\sup _{s \in K_{n}}\left|Y_{a, y}(s)\right|>M_{n}\right) \leq \varepsilon / 2^{n}, \quad n \in \mathbb{N} .
$$

By the compactness principle, the set $H_{\varepsilon}=\left\{f \in H(D): \sup _{s \in K_{n}}|f(s)| \leq\right.$ $\left.M_{n}, n \in \mathbb{N}\right\}$ is compact in $H(D)$, and by (11),

$$
\mathbb{P}\left(Y_{a, y}(s) \in H_{\varepsilon}\right) \geq 1-\varepsilon
$$

for all $a \geq 1$, or

$$
P_{a, y}\left(H_{\varepsilon}\right) \geq 1-\varepsilon
$$

for all $a \geq 1$. So, we have proved that the family $\left\{P_{a, y}\right\}$ is tight, and therefore, by the Prokhorov theorem it is relatively compact.

Moreover, the uniform convergence on compact subsets of $D$ of $\mathcal{Z}_{2, a, y}(s)$ to $\mathcal{Z}_{2, y}(s)$ as $a \rightarrow \infty$ implies

$$
\begin{aligned}
& \lim _{a \rightarrow \infty} \limsup _{N \rightarrow \infty} \mu_{N}\left(\varrho\left(\mathcal{Z}_{2, y}(s+i m h), \mathcal{Z}_{2, a, y}(s+i m h)\right) \geq \varepsilon\right) \\
& \leq \lim _{a \rightarrow \infty} \limsup _{N \rightarrow \infty} \frac{1}{\varepsilon(N+1)} \sum_{m=0}^{N} \varrho\left(\mathcal{Z}_{2, y}(s+i m h), \mathcal{Z}_{2, a, y}(s+i m h)\right)=0 .
\end{aligned}
$$

Hence, setting $Y_{N, y}(s)=\mathcal{Z}_{2, y}\left(s+i \theta_{N}\right)$, we have

$$
\lim _{a \rightarrow \infty} \limsup _{N \rightarrow \infty} \mathbb{P}\left(\varrho\left(Y_{N, a, y}(s), Y_{N, y}(s)\right) \geq \varepsilon\right)=0 .
$$

Since $\left\{P_{a, y}\right\}$ is relatively compact, there is a subsequence $\left\{P_{a_{1}, y}\right\}$ converging weakly to some measure $P_{y}$ on $(H(D), \mathcal{B}(H(D)))$ as $a_{1} \rightarrow \infty$. Now (10) and (12) together with Theorem 4.2 of [1] complete the proof.

5. Approximation of $\mathcal{Z}_{2}(s)$ by $\mathcal{Z}_{2, y}(s)$. In order to pass from the function $\mathcal{Z}_{2, y}(s)$ to $\mathcal{Z}_{2}(s)$ it is sufficient to know that $\mathcal{Z}_{2, y}(s)$ approximates $\mathcal{Z}_{2}(s)$. It turns out that it is sufficient to have an approximation in the mean with respect to the relevant space.

First, we observe that, for $\sigma>1 / 2$,

$$
\mathcal{Z}_{2, y}(s)=\frac{1}{2 \pi i} \int_{\sigma_{1}-i \infty}^{\sigma_{1}+i \infty} \mathcal{Z}_{2}(s+z) l_{y}(z) \frac{d z}{z} .
$$


For $\sigma>1 / 2$ we have $\sigma+\sigma_{1}>1$. Therefore, for $\operatorname{Re} z=\sigma_{1}$,

$$
\mathcal{Z}_{2}(s+z)=\int_{1}^{\infty}|\zeta(1 / 2+i x)|^{4} x^{-s-z} d x .
$$

By the proof of Lemma 8, for $\sigma>1 / 2$,

$$
\begin{aligned}
\int_{1}^{\infty} \frac{a_{y}(x)}{x^{s}} d x & =\frac{1}{2 \pi i} \int_{\sigma_{1}-i \infty}^{\sigma_{1}+i \infty}\left(\frac{l_{y}(z)}{z} \int_{1}^{\infty}|\zeta(1 / 2+i x)|^{4} \frac{d x}{x^{s+z}}\right) d z \\
& =\frac{1}{2 \pi i} \int_{\sigma_{1}-i \infty}^{\sigma_{1}+i \infty} \mathcal{Z}_{2}(s+z) l_{y}(z) \frac{d z}{z}
\end{aligned}
$$

On the other hand, in the proof of Lemma 8 it was shown that the left-hand side of the last equality is $\mathcal{Z}_{2, y}(s)$.

TheORem 11. Let $K$ be a compact subset of the strip $D$. Then

$$
\lim _{y \rightarrow \infty} \limsup _{N \rightarrow \infty} \frac{1}{N+1} \sum_{m=0}^{N} \sup _{s \in K}\left|\mathcal{Z}_{2}(s+i m h)-\mathcal{Z}_{2, y}(s+i m h)\right|=0 .
$$

Proof. In [9] it was proved that, for $\sigma>5 / 6$,

$$
\int_{1}^{T}\left|\mathcal{Z}_{2}(\sigma+i t)\right|^{2} d t=O(T), \quad T \rightarrow \infty .
$$

Together with Cauchy's formula, this leads in the same region to the estimate

$$
\int_{1}^{T}\left|\mathcal{Z}_{2}^{\prime}(\sigma+i t)\right|^{2} d t=O(T), \quad T \rightarrow \infty .
$$

Now the Gallagher lemma (see, for example, [15, Lemma 1.4]) and (14), (15) show that, for $\sigma>5 / 6$ and fixed $m_{0}$,

$$
\begin{aligned}
& \sum_{m=m_{0}}^{N}\left|\mathcal{Z}_{2}(\sigma+i m h)\right|^{2} \leq \frac{1}{h} \int_{m_{0} h}^{N h}\left|\mathcal{Z}_{2}(\sigma+i t)\right|^{2} d t \\
& +\left(\int_{m_{0} h}^{N h}\left|\mathcal{Z}_{2}(\sigma+i t)\right|^{2} d t \int_{m_{0} h}^{N h}\left|\mathcal{Z}_{2}^{\prime}(\sigma+i t)\right|^{2} d t\right)^{1 / 2}=O(N), \quad N \rightarrow \infty .
\end{aligned}
$$

For $1 / 2<\sigma \leq 1$, the function $\mathcal{Z}_{2}(s)$ satisfies the estimate [8]

$$
\mathcal{Z}_{2}(\sigma+i t)=O_{\varepsilon}\left(t^{4(1-\sigma) / 3+\varepsilon}\right), \quad t \geq t_{0}>0 .
$$

Suppose that $5 / 6+\theta_{1} \leq \sigma \leq 1-\theta_{2}, \theta_{1}, \theta_{2}>0$, if $s \in K$. We put $\sigma_{2}=$ 
$5 / 6+\theta_{1} / 2$. Then (13), (17) and the residue theorem yield

$$
\begin{aligned}
\mathcal{Z}_{2, y}(s)= & \frac{1}{2 \pi i} \int_{\sigma_{2}-\sigma-i \infty}^{\sigma_{2}-\sigma+i \infty} \mathcal{Z}_{2}(s+z) l_{y}(z) \frac{d z}{z} \\
& +\mathcal{Z}_{2}(s)+\operatorname{Res}_{z=1-s} \mathcal{Z}_{2}(s+z) l_{y}(z) z^{-1} .
\end{aligned}
$$

Let $L$ be a simple closed contour lying in $D$ and enclosing the set $K$, and let $|L|$ be the length of $L$. Then, using Cauchy's formula, we find that $\sup _{s \in K}\left|\mathcal{Z}_{2}(s+i m h)-\mathcal{Z}_{2, y}(s+i m h)\right| \leq \frac{1}{2 \pi \delta} \int_{L}\left|\mathcal{Z}_{2}(z+i m h)-\mathcal{Z}_{2, y}(z+i m h)\right||d z|$, where $\delta$ is the distance of $L$ from the set $K$. Thus,

$$
\begin{aligned}
& \frac{1}{N+1} \sum_{m=0}^{N} \sup _{s \in K}\left|\mathcal{Z}_{2}(s+i m h)-\mathcal{Z}_{2, y}(s+i m h)\right| \\
& \ll \frac{1}{N \delta} \int_{L}|d z| \sum_{m=0}^{N}\left|\mathcal{Z}_{2}(\operatorname{Re} z+i m h+i \operatorname{Im} z)-\mathcal{Z}_{2, y}(\operatorname{Re} z+i m h+i \operatorname{Im} z)\right| \\
& \ll \frac{|L|}{N \delta} \sup _{s \in L} \sum_{m=0}^{N}\left|\mathcal{Z}_{2}(\sigma+i m h+i t)-\mathcal{Z}_{2, y}(\sigma+i m h+i t)\right| .
\end{aligned}
$$

Let, for brevity,

$$
R_{y}(s)=\operatorname{Res}_{z=1-s} \mathcal{Z}_{2}(s+z) l_{y}(z) z^{-1} .
$$

Then from (18) we derive

Hence

$$
\begin{aligned}
\mathcal{Z}_{2}(\sigma+i m h+i t) & -\mathcal{Z}_{2, y}(\sigma+i m h+i t)=-R_{y}(\sigma+i m h+i t) \\
& +O\left(\int_{-\infty}^{\infty}\left|\mathcal{Z}_{2}\left(\sigma_{2}+i m h+i t+i \tau\right)\right|\left|l_{y}\left(\sigma_{2}-\sigma+i \tau\right)\right| d \tau\right) .
\end{aligned}
$$

$$
\begin{aligned}
& \frac{1}{N} \sum_{m=0}^{N}\left|\mathcal{Z}_{2}(\sigma+i m h+i t)-\mathcal{Z}_{2, y}(\sigma+i m h+i t)\right| \\
& \ll \frac{1}{N} \sum_{m=0}^{N}\left|R_{y}(\sigma+i m h+i t)\right| \\
&+\int_{-\infty}^{\infty}\left|l_{y}\left(\sigma_{2}-\sigma+i \tau\right)\right| \frac{1}{N} \sum_{m=0}^{N}\left|\mathcal{Z}_{2}\left(\sigma_{2}+i m h+i t+i \tau\right)\right| d \tau
\end{aligned}
$$

Since $t$ is bounded, in view of (16) we obtain

$$
\begin{aligned}
& \frac{1}{N} \sum_{m=0}^{N} \mid \mathcal{Z}_{2}\left(\sigma_{2}\right.+i m h+i t+i \tau) \mid \\
& \ll\left(\frac{1}{N} \sum_{m=0}^{N}\left|\mathcal{Z}_{2}\left(\sigma_{2}+i m h+i t+i \tau\right)\right|^{2}\right)^{1 / 2} \ll 1+|\tau| .
\end{aligned}
$$


We have

$$
R_{y}(s)=\lim _{w \rightarrow \infty} \frac{1}{4 !} \frac{d^{4}}{d w^{4}}\left((w-1)^{5} \mathcal{Z}_{2}(w) \frac{1}{\sigma_{1}} \Gamma\left(\frac{w-s}{\sigma_{1}}\right) y^{w-s}\right) .
$$

Therefore, in view of the estimate $\Gamma(s)=O\left(e^{-c|t|}\right), c>0$, valid in any strip $\sigma_{1} \leq \sigma \leq \sigma_{2}$, an application of the Gallagher lemma again yields

$$
\frac{1}{N} \sum_{m=0}^{N}\left|R_{y}(\sigma+i m h+i t)\right|=o(1), \quad N \rightarrow \infty .
$$

Suppose that, for $s \in L, \sigma \geq 5 / 6+3 \theta_{1} / 4$ and $\delta \geq \theta_{1} / 4$. Then (19)-(22) show that

$$
\begin{aligned}
& \frac{1}{N+1} \sum_{m=0}^{N} \sup _{s \in K}\left|\mathcal{Z}_{2}(s+i m h)-\mathcal{Z}_{2, y}(s+i m h)\right| \\
& \ll \sup _{s \in L} \int_{-\infty}^{\infty}\left|l_{y}\left(\sigma_{2}-\sigma+i \tau\right)\right|(1+|\tau|) d \tau+o(1) \\
& \ll \sup _{\sigma<-\theta_{1} / 4} \int_{-\infty}^{\infty}\left|l_{y}(\sigma+i t)\right|(1+|t|) d t+o(1)
\end{aligned}
$$

as $N \rightarrow \infty$. However,

$$
\lim _{y \rightarrow \infty} \sup _{\sigma<-\theta_{1} / 4} \int_{-\infty}^{\infty}\left|l_{y}(\sigma+i t)\right|(1+|t|) d t=0,
$$

and the theorem follows from (23).

\section{Proof of the main theorems}

Proof of Theorem 3. First we observe that the family $\left\{P_{\sigma, y}\right\}$ is tight, where $P_{\sigma, y}$ is the limit measure in Theorem 9 . Indeed, let $X_{y}(\sigma)$ be a complex-valued random variable with distribution $P_{\sigma, y}$. Then by Theorem 9 we have

$$
X_{N, y}(\sigma) \underset{N \rightarrow \infty}{\stackrel{\mathcal{D}}{\longrightarrow}} X_{y}(\sigma) .
$$

In view of Theorem 11 and (16) we find that, for $\sigma>5 / 6$,

$$
\sup _{y \geq 1} \limsup _{N \rightarrow \infty} \frac{1}{N+1} \sum_{m=0}^{N}\left|\mathcal{Z}_{2, y}(\sigma+i m h)\right| \leq R<\infty .
$$

Therefore, taking $M=R \varepsilon^{-1}$ and using arguments similar to those in the proof of Theorem 9, we deduce that

$$
\limsup _{N \rightarrow \infty} \mathbb{P}\left(\left|X_{N, y}(\sigma)\right|>M\right) \leq \varepsilon .
$$

Hence $P_{\sigma, y}\left(C_{\varepsilon}\right) \geq 1-\varepsilon$ for all $y \geq 1$, i.e., $\left\{P_{\sigma, y}\right\}$ is tight. Here we preserve the notation of Section 4 . 
Now let $X_{N}(\sigma)=\mathcal{Z}_{2}\left(\sigma+i \theta_{N}\right)$. By Theorem 11,

$$
\begin{aligned}
& \lim _{y \rightarrow \infty} \limsup _{N \rightarrow \infty} \mu_{N}\left(\left|\mathcal{Z}_{2}(\sigma+i m h)-\mathcal{Z}_{2, y}(\sigma+i m h)\right| \geq \varepsilon\right) \\
& \quad \leq \lim _{y \rightarrow \infty} \limsup _{N \rightarrow \infty} \frac{1}{\varepsilon(N+1)} \sum_{m=0}^{N}\left|\mathcal{Z}_{2}(\sigma+i m h)-\mathcal{Z}_{2, y}(\sigma+i m h)\right|=0 .
\end{aligned}
$$

Therefore,

$$
\lim _{y \rightarrow \infty} \limsup _{N \rightarrow \infty} \mathbb{P}\left(\left|X_{N}(\sigma)-X_{N, y}(\sigma)\right| \geq \varepsilon\right)=0 .
$$

Since the family $\left\{P_{\sigma, y}\right\}$ is tight, it is relatively compact. Let $y_{1}$ be a subsequence of $y$ such that $P_{\sigma, y_{1}}$ converges weakly, say to $P_{\sigma}$, as $y_{1} \rightarrow \infty$. Then

$$
X_{y_{1}}(\sigma) \underset{y_{1} \rightarrow \infty}{\stackrel{\mathcal{D}}{\longrightarrow}} P_{\sigma} .
$$

Now (24) and (25) show that Theorem 4.2 of [1] can be applied, and we obtain

$$
X_{N}(\sigma) \underset{N \rightarrow \infty}{\stackrel{\mathcal{D}}{\longrightarrow}} P_{\sigma}
$$

The theorem is proved.

Proof of Theorem 4. Let $Y_{y}(s)$ be an $H(D)$-valued random element with distribution $P_{y}$. Hereafter, we keep the notation used in the proof of Theorem 10. By that theorem,

$$
Y_{N, y}(s) \underset{N \rightarrow \infty}{\stackrel{\mathcal{D}}{\longrightarrow}} Y_{y}(s) .
$$

The properties of $\mathcal{Z}_{2, y}(s)$ and Theorem 9 show that

$$
\sup _{y \geq 1} \limsup _{N \rightarrow \infty} \frac{1}{N+1} \sum_{m=0}^{N} \sup _{s \in K_{n}}\left|\mathcal{Z}_{2, y}(s+i m h)\right| \leq R_{n}<\infty .
$$

Hence, similarly to the proof of Theorem 10 we infer that $\left\{P_{y}\right\}$ is tight. Moreover, Theorem 11 implies

$$
\begin{aligned}
& \lim _{n \rightarrow \infty} \limsup _{N \rightarrow \infty} \mu_{N}\left(\varrho\left(\mathcal{Z}_{2}(s+i m h), \mathcal{Z}_{2, y}(s+i m h)\right) \geq \varepsilon\right) \\
& \quad \leq \lim _{y \rightarrow \infty} \limsup _{N \rightarrow \infty} \frac{1}{\varepsilon(N+1)} \sum_{m=0}^{\infty} \varrho\left(\mathcal{Z}_{2}(s+i m h), \mathcal{Z}_{2, y}(s+i m h)\right)=0 .
\end{aligned}
$$

Hence, taking $Y_{N}(s)=\mathcal{Z}_{2}\left(s+i \theta_{N}\right)$, we find that

$$
\lim _{y \rightarrow \infty} \limsup _{N \rightarrow \infty} \mathbb{P}\left(\varrho\left(Y_{N}(s), Y_{N, y}(s)\right) \geq \varepsilon\right)=0 .
$$

The tightness of $\left\{P_{y}\right\}$ gives its relative compactness. Let $y_{1}$ be a subsequence 
of $y$ such that $P_{y_{1}}$ converges weakly, say to $P$, as $y_{1} \rightarrow \infty$. Then

$$
Y_{y_{1}} \underset{y_{1} \rightarrow \infty}{\stackrel{\mathcal{D}}{\longrightarrow}} P
$$

and the theorem is a consequence of (26)-(28) and Theorem 4.2 of [1].

\section{References}

[1] P. Billingsley, Convergence of Probability Measures, Wiley, New York, 1968.

[2] K. M. Bitar, N. N. Kim and H. C. Ren, Path integrals and Voronin's theorem on the universality of the Riemann zeta-function, Ann. Phys. 211 (1991), 172-196.

[3] H. Bohr und B. Jessen, Über die Wertverteilung der Riemannschen Zetafunktion, Erste Mitteilung, Acta Math. 54 (1930), 1-35.

[4] -, -, Über die Wertverteilung der Riemannschen Zetafunktion, Zweite Mitteilung, ibid. 58 (1932), 1-55.

[5] J. B. Conway, Functions of One Complex Variable, Springer, New York, 1973.

[6] H. Heyer, Probability Measures on Locally Compact Groups, Springer, Berlin, 1977.

[7] A. Ivić, On some conjectures and results for the Riemann zeta-function and Hecke series, Acta Arith. 99 (2001), 115-145.

[8] - On the estimation of $\mathcal{Z}_{2}(s)$, in: Analytic and Probabilistic Methods in Number Theory, A. Dubickas et al. (eds.), TEV, Vilnius, 2002, 83-98.

[9] -, On the estimation of some Mellin transforms connected with the fourth moment of $|\zeta(1 / 2+i t)|$, in: Elementare und Analytische Zahlentheorie, W. Schwarz and J. Steuding (eds.), Steiner, Stuttgart, 2006, 77-88.

[10] A. Ivić, M. Jutila and Y. Motohashi, The Mellin transform of powers of the zetafunction, Acta Arith. 95 (2002), 305-342.

[11] M. Jutila, The Mellin transform of the fourth power of Riemann's zeta-function, in: Number Theory (Chennai, 2004), Ramanujan Math. Soc., Mysore, 2005, 15-29.

[12] A. Laurinčikas, Limit Theorems for the Riemann Zeta-Function, Kluwer, Dordrecht, 1996.

[13] - Limit theorems for the Mellin transforms of $|\zeta(1 / 2+i t)|^{4}$, submitted.

[14] K. Matsumoto, Probabilistic value-distribution theory of zeta-functions, Sugaku Expositions 17 (2004), 51-71.

[15] H. L. Montgomery, Topics in Multiplicative Number Theory, Springer, Berlin, 1971.

[16] K. Yosida, Functional Analysis, Springer, Berlin, 1971.

V. Balinskaitè and A. Laurinčikas

Department of Mathematics and Informatics Institute of Mathematics and Informatics Vilnius University

Naugarduko 24

03225 Vilnius, Lithuania

E-mail: antanas.laurincikas@maf.vu.lt
A. Laurinčikas Akademijos 4 08663 Vilnius, Lithuania

Received on 24.5.2006

and in revised form on 29.10.2007 